\title{
Identification and validation of a commercial cryopreservation medium for the practical preservation of Dirofilaria immitis microfilaria
}

Takahiro Shirozu, Akira Soga and Shinya Fukumoto*

\begin{abstract}
Background: Dirofilaria immitis is a parasitic nematode transmitted by mosquitoes and the cause of heartworm disease in dogs and dirofilariasis in humans and other mammals. The parasite is endemic worldwide. Vector stage research requires a reliable supply of $D$. immitis microfilariae $(\mathrm{mf})$. It is believed that cryopreserved $\mathrm{mf}$ would retain viability and provide a powerful tool for vector stage research. However, reports on cryopreservation of D. immitis mf are limited. Therefore, this study aimed to validate commercial cryopreservation media to establish a practical, convenient and reproducible storage procedure for D. immitis $\mathrm{mf}$.
\end{abstract}

Methods: Six different commercially available cryopreservation media were compared with the traditional polyvinylpyrrolidone-dimethyl sulfoxide (PVP-DMSO) preservation solution. In vitro viability of purified D. immitis mf and mf-infected total blood was analyzed using a motility assay and propidium iodide staining. In vivo infectivity of Aedes aegypti mosquitoes with cryopreserved $\mathrm{mf}$ was assessed using a mosquito survival test and quantifying the number of third-stage larvae ( $L 3$ ) after 13 days post-infection.

Results: Purified $\mathrm{mf}$ cryopreserved in CultureSure showed the best viability when compared to $\mathrm{mf}$ cryopreserved in the remaining five commercially available media and PVP-DMSO. Viability of $\mathrm{mf}$ in mf-infected total blood cryopreserved in CultureSure varied with the ratio of infected blood to CultureSure. Optimum results were obtained with $200 \mu \mathrm{lmf}$-infected blood:800 $\mu \mathrm{l}$ CultureSure. CultureSure was also the optimum medium for cryopreserving mf prior to infectivity of A. aegypti. The number of $\mathrm{L} 3$ was approximately the same for CultureSure cryopreserved mf (3x concentrated solution) and non-cryopreserved fresh $\mathrm{mf}$.

Conclusions: CultureSure is an optimal commercial cryopreservation solution for the storage of D. immitis purified $\mathrm{mf}$, mf-infected total blood, and $\mathrm{mf}$ used for in vivo mosquito experiments. Furthermore, this study describes an easy preservation method for clinical D. immitis-infected blood samples facilitating vector stage studies, as well as the study of macrocyclic lactone resistance in heartworms and the education of veterinarians.

Keywords: Aedes aegypti, Cryopreservation media, CultureSure, Dirofilaria immitis, Heartworm disease, Parasitic nematode, Microfilariae

*Correspondence: fukumoto@obihiro.ac.jp

National Research Center for Protozoan Diseases, Obihiro University of Agriculture and Veterinary Medicine, Obihiro, Hokkaido 080-8555, Japan

(C) The Author(s) 2020. This article is licensed under a Creative Commons Attribution 4.0 International License, which permits use, sharing, adaptation, distribution and reproduction in any medium or format, as long as you give appropriate credit to the original author(s) and the source, provide a link to the Creative Commons licence, and indicate if changes were made. The images or other third party material in this article are included in the article's Creative Commons licence, unless indicated otherwise in a credit line to the material. If material is not included in the article's Creative Commons licence and your intended use is not permitted by statutory regulation or exceeds the permitted use, you will need to obtain permission directly from the copyright holder. To view a copy of this licence, visit http://creativecommons.org/licenses/by/4.0/. The Creative Commons Public Domain Dedication waiver (http://creativecommons.org/publicdomain/zero/1.0/) applies to the data made available in this article, unless otherwise stated in a credit line to the data. 


\section{Background}

The parasitic nematode Dirofilaria immitis is transmitted by mosquitoes causing potentially lethal heartworm disease in dogs, pulmonary dirofilariasis in humans, and dirofilariasis in various mammals [1]. Wild carnivores are also sentinels for the spread of $D$. immitis. Despite the availability of effective preventive medicines, $D$. immitis is endemic worldwide. Therefore, a better understanding of host-parasite interactions between mosquito vectors and $D$. immitis is needed to establish a truly effective control strategy.

Vector stage experiments require live $D$. immitis microfilariae (mf) obtained from infected animals [2]. Having a reliable supply of live $\mathrm{mf}$ on demand is very important. Cryopreservation is a powerful tool allowing pathogens to be stored in a viable state. However, there are only a few reports describing cryopreservation of $D$. immitis mf. Initially, in 1947, Weinman and McAllister [3] demonstrated cryopreservation of $\mathrm{mf}$ in citrated or heparinized blood without cryoprotectant additives. Taylor [4] in 1960 and Lok et al. [5] in 1983 used glycerol and dimethylsulfoxide (DMSO), respectively, for cryopreservation of $\mathrm{mf}$ and assessed the subsequent infectivity of mosquitoes. In the most recent study, published in 2001, Bartholomay et al. [6] prepared and tested a $16 \%$ polyvinylpyrrolidone (PVP)-6\% DMSO solution (PVP-DMSO) as a cryoprotective medium.

In the previous studies, the cryopreservation media tested were originally prepared by the investigators. Recently, various commercial cryopreservatives have become available and they are commonly used, especially for mammalian cell cryopreservation. For convenience, reproducibility and standardization purposes, we sought to identify a commercially available cryopreservation medium for effective $D$. immitis mf preservation. To this end, here, we compared six commercial cryopreservation media with the traditional PVP-DMSO preservative [6] for the cryopreservation of D. immitis $\mathrm{mf}$. Effectiveness was evaluated by measuring in vitro viability and in vivo infectivity of mosquitoes.

\section{Methods}

\section{Animals}

Female beagles (2-4 years-old) were experimentally infected with $D$. immitis SF1 strain originally isolated in Japan [7] and reared under a veterinarian's administration. Aedes aegypti Liverpool (LVP)-OB strain, kindly provided by Dr R Maeda, the Obihiro University of Agriculture and Veterinary Medicine (OUAVM), Japan, susceptible to $D$. immitis infection was maintained at $27{ }^{\circ} \mathrm{C}$ and $>80 \%$ humidity on a diet of $5 \%$ fructose and a 12:12 h light:dark photoperiod.

\section{Microfilariae purification and cryopreservation}

Cryopreservation was initially tested using purified $D$. immitis $\mathrm{mf}$ isolated from heparinized blood of an experimentally infected dog. The $\mathrm{mf}$ were purified using a PD-10 gel purification column (GE Healthcare, Amersham Place, UK) equilibrated with RPMI 1640 Medium (Gibco 2120429, Thermo Fisher Scientific, Waltham, MA, USA) supplemented with $1 \times$ penicillin-streptomycin (Gibco 15140122) [8, 9]. Purified mf were centrifuged at $1000 \times g$ for $10 \mathrm{~min}$ at room temperature. The supernatant was discarded and the pelleted $\mathrm{mf}$ were resuspended in $1 \mathrm{ml}$ of cryopreservation medium (c.3000 or $15,000 \mathrm{mf} /$ tube) and transferred to cryogenic tubes (Nunc 119546, Thermo Fisher Scientific). Samples were stored in freezing containers $\left(\right.$ Corning $^{\circledR}$ CoolCell $^{\circledR} \mathrm{LX}$, Corning, NY, USA) overnight at $-80{ }^{\circ} \mathrm{C}$ before being transferred into liquid nitrogen, and stored until analysis. Cryopreservation media used in this study were as follows: PVP-DMSO (0.004 M PVP, average molecular weight of 40,000 and 6\% DMSO) in phosphate buffered saline (PBS) $[6,10]$, CultureSure ${ }^{\circledR}$ Freezing Medium (CultureSure, FUJIFILM Wako, Osaka, Japan), Cellbanker ${ }^{\circledR} 1$ (Cellbanker, Zenoaq Resource, Shiga, Japan), LaboBanker 1 (LaboBanker, Kurabo, Osaka, Japan), Cos banker (Cosmo Bio, Tokyo, Japan), Bambanker ${ }^{\circledR}$ (GC Lymphotec, Tokyo, Japan), CryoScarless ${ }^{\circledR}$ DMSO-Free (CryoScarless, Bio Verde, Kyoto, Japan), and RPMI 1640 (Table 1).

\section{In vitro motility assay}

Cryopreserved $\mathrm{mf}$ were thawed rapidly by swirling in a water bath at $37{ }^{\circ} \mathrm{C}$. Immediately after thawing, the $\mathrm{mf}$ were suspended in $10 \mathrm{ml}$ of RPMI 1640 medium and centrifuged at $1500 \times g$ for $10 \mathrm{~min}$ at room temperature. The pelleted $\mathrm{mf}$ were re-suspended in $1 \mathrm{ml}$ of RPMI 1640 medium containing $5 \%$ fetal calf serum, transferred to 12-well cell culture plates (Nunc) and incubated in 5\% $\mathrm{CO}_{2}$ in air at $37{ }^{\circ} \mathrm{C}$ for 3 days. The motility of $\mathrm{mf}$ was assessed microscopically on days 0,1 and 3 post-thawing (dpt) $(n \geq 3)$ and subjectively scored as inactive or dead $(-)$, less active $(+)$, moderately active $(++)$ and highly active $(+++)[11]$. The results were statistically analyzed to rank the various media using a Chi-square test and adjusted residuals (AR). Absolute AR values were considered significant at $A R \geq 1.96$. The difference between PVP-DMSO and each cryopreservation medium was analyzed using Welch's t-test.

\section{Cryopreservation of $D$. immitis mf-infected total blood}

Fresh, heparinized blood (50-400 $\mu \mathrm{l})$ was collected from D. immitis-infected dogs $(c .25,000 \mathrm{mf} / \mathrm{ml})$ and mixed with CultureSure up to $1 \mathrm{ml}$ and then cryopreserved as described above. Differences in motility between purified 
$\mathrm{mf}$ and $\mathrm{mf}$-infected total blood were analyzed by one-way analysis of variance (ANOVA), followed by Tukey's multiple comparisons test.

\section{Propidium iodide $(\mathrm{PI})$ staining assay}

To evaluate any physical damage caused by cryopreservation, cultured $\mathrm{mf}$ were stained with PI $(2 \mu \mathrm{g} / \mathrm{ml})$ (Molecular probes, Eugene, OR, USA) for $10 \mathrm{~min}$ on $3 \mathrm{dpt}$. The stained $\mathrm{mf}$ were washed with PBS by centrifugation at $1500 \times g$ for $10 \mathrm{~min}$ at $4{ }^{\circ} \mathrm{C}$ and then subjected to fluorescence microscopy observation using the same exposure time in each experiment. Methanol-fixed $\mathrm{mf}$ were used as a positive control. The results were statistically compared using a Chi-square test and calculated AR.

\section{In vivo third-stage larva (L3) development assay}

Mf cryopreserved with commercial CultureSure, LaboBanker media and traditional PVP-DMSO were used to determine the infectivity of mosquitoes in vivo. Thawed $\mathrm{mf}$ were fed to female $A$. aegypti on day 7 postemergence for $2 \mathrm{~h}$ by artificial membrane feeding using Parafilm (Bemis company, Neenah, WI, USA). Bloodfeeding experiments were completed within $4 \mathrm{~h}$ postthawing. The concentration of thawed $\mathrm{mf}$ was adjusted to $18 \mathrm{mf} / \mu \mathrm{l}$ using heparinized blood collected from a healthy dog. Non-cryopreserved fresh blood $(6 \mathrm{mf} /$ $\mu \mathrm{l})$ was collected from an infected dog and used as the control. Fully fed mosquitoes were selected under $\mathrm{CO}_{2}$ anesthesia and maintained for $13 \mathrm{~d}$. Mosquito survival was monitored every day. At day 13 post-infection (dpi), all surviving female mosquitoes were dissected under a stereomicroscope to investigate the number of L3. Mosquito survival was analyzed by Chi-square test and the L3 count was analyzed by Welch's t-test.

\section{Statistical analyses}

All statistical analyses were performed using GraphPad Prism 7 (GraphPad, San Diego, CA, USA). A significant difference was defined by $P<0.05$ and $P<0.10$ was considered a marginal difference.

\section{Results and discussion}

\section{In vitro viability of purified microfilariae}

The viability of cryopreserved mf was compared between traditional PVP-DMSO and several commercially available cryopreservation media. Microscopic observation revealed that $\mathrm{mf}$ cryopreserved with CultureSure exhibited the highest motility $\left(\chi^{2}=721.2, d f=7, P<0.0001, \mathrm{AR}=8.5\right.$ on 0 $\mathrm{dpt} ; \chi^{2}=648.5, d f=7, P<0.0001, \mathrm{AR}=10.51$ on $1 \mathrm{dpt}$ and $\chi^{2}=333.3, d f=7, P<0.0001, \mathrm{AR}=12.19$ on $3 \mathrm{dpt}$ ) (Fig. 1a). Mf cryopreserved with LaboBanker exhibited the second highest motility ( $\mathrm{AR}=7.67$ on $0 \mathrm{dpt}, 9.88$ on $1 \mathrm{dpt}$, and 6.55 on $3 \mathrm{dpt}$ ), and Mf in CryoScarless exhibited the lowest motility $(\mathrm{AR}=-24.03$ on $0 \mathrm{dpt},-20.06$ on $1 \mathrm{dpt}$, and -10.12 on $3 \mathrm{dpt}$ ). When mf motility was compared based on moderately active and highly active levels, $\mathrm{mf}$ in CultureSure $(t=6.634, d f=2.214, P=0.017$ on $0 \mathrm{dpt} ; t=6.682$, $d f=3.118, P=0.0061$ on $1 \mathrm{dpt}$ and $t=6.76, d f=3.835$, $P=0.0029$ on $3 \mathrm{dpt})$, LaboBanker $(t=4.495, d f=3.934$, $P=0.011$ on $0 \mathrm{dpt} ; t=5.944, d f=3.373, P=0.0068$ on 1 dpt and $t=4.493, d f=3.997, P=0.011$ on $3 \mathrm{dpt}$ ) and Cellbanker $(t=5.024, d f=3.571, P=0.0099$ on $0 \mathrm{dpt}$; $t=6.184$, $d f=2.383, P=0.016$ on $1 \mathrm{dpt}$ and $t=3.02, d f=3.698$, $P=0.043$ on $3 \mathrm{dpt}$ ) media all exhibited significantly higher motility on 0,1 and $3 \mathrm{dpt}$ than $\mathrm{mf}$ preserved in traditional PVP-DMSO media. Mf in CryoScarless medium showed significantly lower motility compared to PVP-DMSO on $0(t=18.87, d f=2.116, P=0.0022)$ and $1 \mathrm{dpt}(t=10.61$, $d f=2.071, P=0.0078)$.

The viability of $\mathrm{mf}$ on $3 \mathrm{dpt}$ was quantified based on the ability of PI to penetrate dead or dying cells but not living cells. CultureSure-cryopreserved $\mathrm{mf}$ showed significantly lower PI values compared to all of the other cryopreservation media $\left(\chi^{2}=161.3, d f=7, P<0.0001\right.$, $\mathrm{AR}=5.7$ ) (Fig. 1b). The $\mathrm{mf}$ cryopreserved in PVPDMSO $\quad(\mathrm{AR}=3.59), \quad$ LaboBanker $\quad(\mathrm{AR}=2.87) \quad$ and Bambanker $(A R=2.53)$ showed significantly lower PI positive rates (Fig. 1b). These results are supported by fluorescent imaging of $\mathrm{mf}$ PI staining in each medium (Fig. 1c). Translating our results, to do a parallel with other published studies, the total motile parasite ratio

Table 1 Information about the composition of commercial cryopreservation media

\begin{tabular}{llll}
\hline Product name & DMSO (\%) & Bovine serum & Manufacturer \\
\hline Cellbanker 1 & 10 & $<80 \%$ & Zenoaq Resource \\
LaboBanker 1 & 10 & Containing & Kurabo \\
CultureSure Freezing Medium & 10 & Free (containing BSA) & Fujifilm \\
Bambanker & 10 & Free (containing bovine serum-derived & GC Lymphotec \\
Cos banker & Containing & components) & Cosmo Bio \\
CryoScarless DMSO-Free & Free & Free & Bio Verde \\
\hline
\end{tabular}

a The concentration is not stated by the manufacturers 
of CultureSure-cryopreserved $\mathrm{mf}$ after thawing (97.2\%, Fig. 1a) was considered equivalent or slightly better than the reported for cryopreserved $\mathrm{mf}$ using 5\% DMSO medium (78.8-91.6\%) [5] or PVP-DMSO (>95\%) [6]. From these experiments, it is clear that CultureSure is the optimal cryopreservation medium tested for the preservation of $D$. immitis $\mathrm{mf}$.

Parasite concentration levels were a factor influencing motility in the various media. In general, motility of mf cryopreserved at a high concentration $(c .15,000 \mathrm{mf} / \mathrm{ml})$ was significantly greater than $\mathrm{mf}$ cryopreserved at a low concentration $(c .3000 \mathrm{mf} / \mathrm{ml})$. This was true for $\mathrm{mf}$ cryopreserved in PVP-DMSO on 0 to $3 \mathrm{dpt}(t=4.451, d f=3.499$, $P=0.015$ on $0 \mathrm{dpt} ; t=36.17, d f=2.929, P<0.0001$ on $1 \mathrm{dpt}$ and $t=11, d f=2.414, P=0.004$ on $3 \mathrm{dpt}$ ), in CultureSure on $1(t=4.708, d f=3.817, P=0.010)$ and $3 \mathrm{dpt}(t=3.805$, $d f=2.865, P=0.035)$ and in LaboBanker on $0(t=4.748$, $d f=3.291, P=0.014)$ and $3 \mathrm{dpt}(t=6.522, d f=2.901$, $P=0.008$ ) (Fig. 1d). These results suggest that cryopreservation of $\mathrm{mf}$ should be carried out at the higher $\mathrm{mf}$ concentration to retain better viability.

\section{In vitro viability of microfilariae in mf-infected total blood} Cryopreservation of $D$. immitis mf-infected total blood is especially important in clinical settings. The motility of $\mathrm{mf}$ in $\mathrm{mf}$-infected total blood was compared over a range of blood volumes in CultureSure (1 $\mathrm{ml}$ total volume) and with purified $\mathrm{mf}(c .25,000 \mathrm{mf} / \mathrm{ml})$ (Fig. 2a). The results were comparable to purified mf cryopreservation, in most cases. Significant differences were observed between purified $\mathrm{mf}$ and mf-infected total blood volumes of $400 \mu \mathrm{l}$ on $0 \mathrm{dpt}$ $(q=5.431, d f=12, P=0.022)$ and $300 \mu \mathrm{l}$ on $3 \mathrm{dpt}(q=5.38$, $d f=12, P=0.024)$. In addition, a significant difference was seen for mf-infected volumes of $200 \mu \mathrm{l}$ and $400 \mu \mathrm{l}$ on 0 dpt ( $q=5.048, d f=12, P=0.035)$. Analysis of PI staining of $\mathrm{mf}$ on $3 \mathrm{dpt}$, demonstrated that mf-infected total blood at a volume of $200 \mu \mathrm{l}$ showed significantly lower PI positive values $\left(\chi^{2}=12.19, d f=5, P=0.032\right.$, AR $=2.36$, Fig. $\left.2 \mathrm{~b}\right)$, whereas the $300 \mu \mathrm{l}$ sample showed the highest PI staining values $(\mathrm{AR}=1.98$, Fig. $2 \mathrm{~b})$. These findings were supported by fluorescent imaging (Fig. 2c). Altogether, these results suggest that cryopreservation is optimal with $200 \mu \mathrm{l}(c .25$ $\mathrm{mf} / \mu \mathrm{l}$ ) of infected blood brought to a total volume of $1 \mathrm{ml}$ with CultureSure.

\section{Vector infectivity and survival of cryopreserved microfilariae}

Infectivity of mosquito vectors with $D$. immitis mf cryopreserved with CultureSure or LaboBanker was compared with traditional PVP-DMSO cryopreservation. Non-cryopreserved, fresh $\mathrm{mf}$ were used as a positive control. The survival rate of mosquitoes fed LaboBanker cryopreserved $\mathrm{mf}$ was significantly higher compared to mosquitoes fed
CultureSure $\left(x^{2}=4.761, d f=1, P=0.029\right)$ or PVP-DMSO $\left(x^{2}=4.086, d f=1, P=0.043\right)$ cryopreserved $\mathrm{mf}$ on $13 \mathrm{dpi}$ (Fig. 3a). The number of L3 in mosquitoes fed CultureSure cryopreserved $\mathrm{mf}$ tended to be higher than that of the L3 for $\mathrm{mf}$ cryopreserved in PVP-DMSO $(t=1.728, d f=53.64$, $P=0.090)$ and fresh $(t=1.897, d f=56.14, P=0.063) \mathrm{mf}$ (Fig. 3b). Although different mosquito colonies and $\mathrm{mf}$ infectious inocula were used, the $\mathrm{mf}$ infectivity reported here was similar to the one previously reported for PVPDMSO cryopreserved $\mathrm{mf}$ [6]. The ratios of mean L3 numbers in individual mosquitoes/feeding inoculum concentration were 0.85 and 0.77 for PVP-DMSO cryopreserved $\mathrm{mf}$ in our study and a previous study, respectively [6]. Remarkably, this ratio, determined for CultureSure was 1.03. The number of L3 of mosquitoes fed LaboBanker cryopreserved $\mathrm{mf}$ was significantly lower compared to the other treatments (Fresh: $t=5.868, d f=71.41, P<0.0001$; PVP-DMSO: $t=5.67, d f=45, P<0.0001$; and CultureSure: $t=6.496, d f=42.96, P<0.0001)$. These results indicate that CultureSure is an optimal medium for $D$. immitis mf cryopreservation. Lok et al. [5] demonstrated that $6.0-20.0 \%$ of $\mathrm{mf}$ cryopreserved in 5\% DMSO medium reached the L3 stage when inoculated to mosquitoes using the enema method. The estimated average blood volume ingested by A. aegypti per blood meal was $2.3-2.6 \mu \mathrm{l}[12,13]$. In our study, assuming a blood intake of $2.5 \mu \mathrm{l}(18 \mathrm{mf} / \mu \mathrm{l})$ per mosquito, the L3 development ratio was calculated as $41.8 \%$ (18.8 L3/45 mf), suggesting CultureSure is better than 5\% DMSO medium for the cryopreservation of D. immits $\mathrm{mf}$.

\section{Importance of DMSO in cryopreservation media}

DMSO appears to be an important component in cryopreservation media for $D$. immitis $\mathrm{mf}$. In the present study, most of the commercial cryopreservation media examined yielded a positive performance, except for CryoScarless, which was the only medium that did not contain DMSO. DMSO is known to inhibit ice crystal formation during the freezing process and it is commonly used for mammalian cell cryopreservation [14]. Selection of a DMSO-containing medium seems critical for successful cryopreservation of D. immitis mf.

\section{Justification and practical applications for using cryopreserved microfilariae}

Preparation of D. immitis-infected animals and fresh $\mathrm{mf}$ is a bottleneck for in vivo mosquito vector experiments. Here, we demonstrated that cryopreserved $\mathrm{mf}$ could be used for in vivo mosquito experiments instead of fresh $\mathrm{mf}$, thereby eliminating the need to maintain infected animals. The concentration of cryopreserved $\mathrm{mf}$ fed to mosquitoes in mf-infected blood was adjusted to 3-fold higher levels than non-cryopreserved, fresh mf. Under these conditions, the 


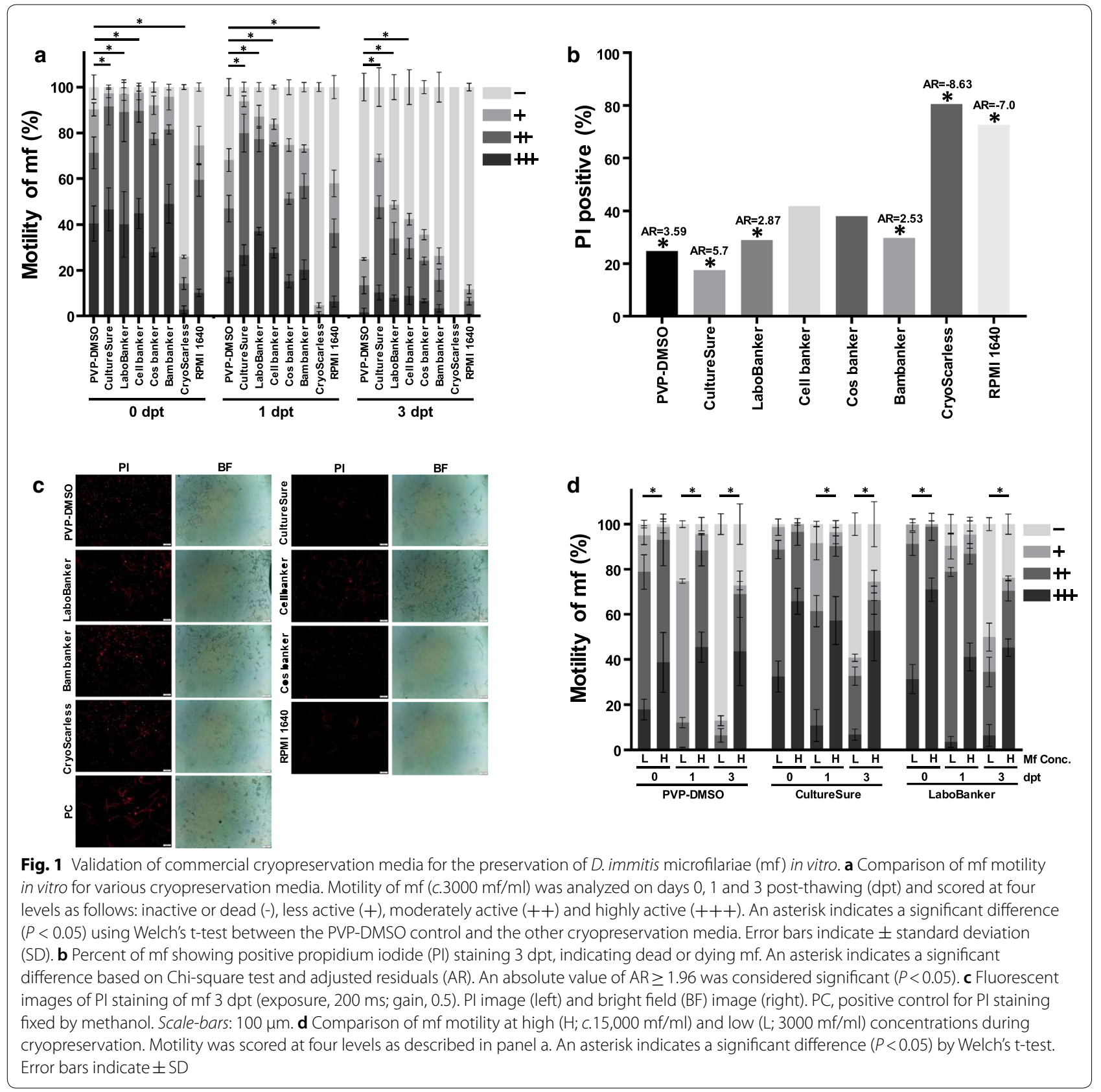

number of L3 for the mosquitoes was almost equivalent to that of fresh $\mathrm{mf}$.

Standardization of $D$. immitis $\mathrm{mf}$ cryopreservation method is essential for the study of drug-resistant $D$. immitis. Macrocyclic lactones have been widely used to prevent heartworm infections for the past three decades [15]. Since the emergence of macrocyclic lactone resistance in D. immitis in 2005 [16], numerous field and laboratoryinduced isolates have been studied [17-19]. These isolates are essential for further studies to completely understand drug resistance in D. immitis. However, it is quite expensive and time-consuming to maintain each of these isolates by continuous alternate passages in mosquitoes and dogs. Although cryopreservation methods are not without limitations, such as the need for continuous and relatively frequent replenishment of liquid nitrogen levels, they offer unique advantages over continuous biological maintenance. In addition and importantly, cryopreservation allows the reduction of animal use (fewer dogs are needed), respecting the ethics in animal experimentation, and makes 

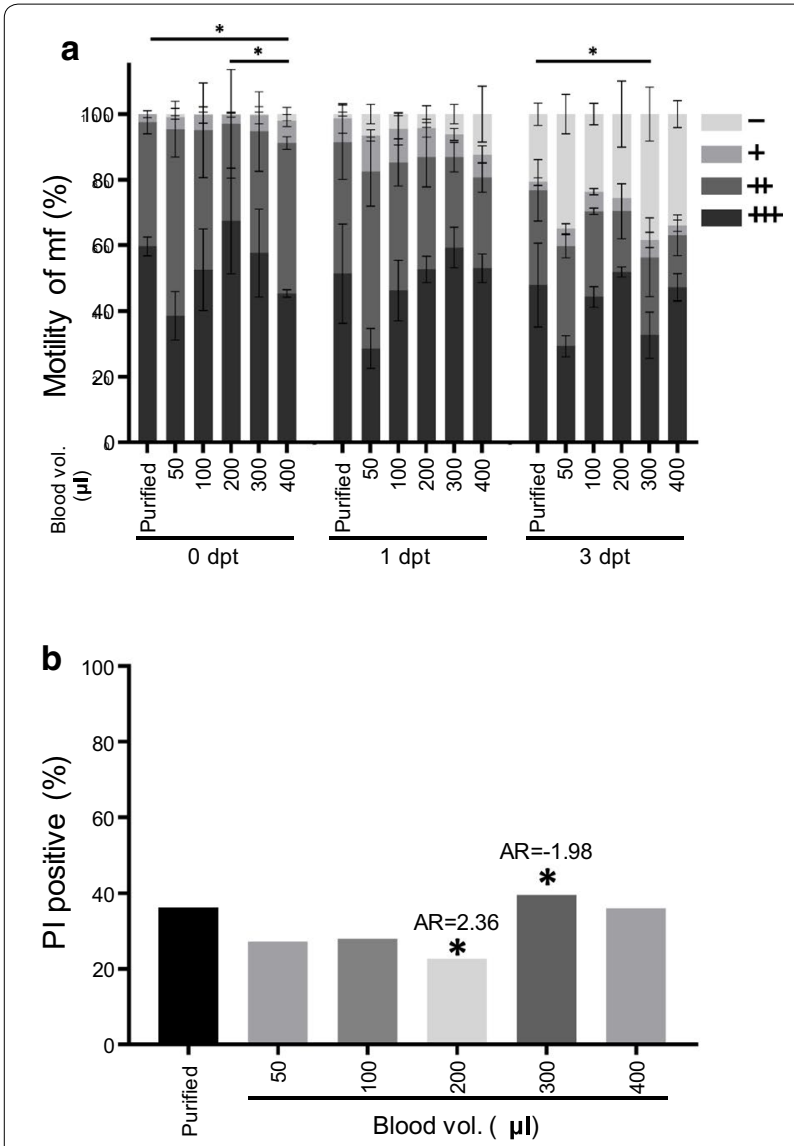

C
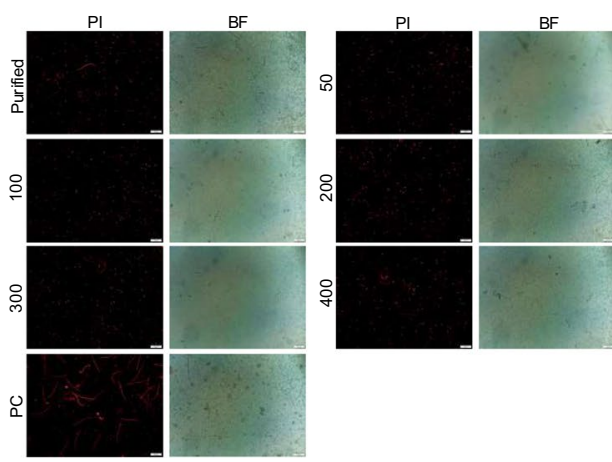

Fig. 2 Viability of cryopreserved D. immitis microfilariae (mf)-infected total blood. a In vitro motility was scored over a range of blood volumes (X-axis) in CultureSure (1 $\mathrm{ml}$ total volume) and compared to purified $\mathrm{mf}$ at 0,1 and 3 days post-thawing (dpt). Scoring was at four levels as follows: inactive or dead $(-)$, less active $(+)$, moderately active $(++)$ and highly active $(+++)$. An asterisk indicates a significant difference $(P<0.05)$ by one-way ANOVA, followed by Tukey's multiple comparisons test. Error bars indicate $\pm S D$. b Percent positive PI staining of mf-infected total blood on $3 \mathrm{dpt}$. An asterisk indicates a significant difference by Chi-square test and adjusted residual (AR). $A R \geq 1.96$ was considered significant $(P<0.05)$. $\mathbf{c}$ Fluorescent images of propidium iodide (PI) staining of mf-infected total blood on 3 dpt (exposure, 100 ms; gain, 0.5). Pl image (left) and bright field (BF) image (right). Abbreviation: PC, positive control. Scale-bars: $100 \mu \mathrm{m}$
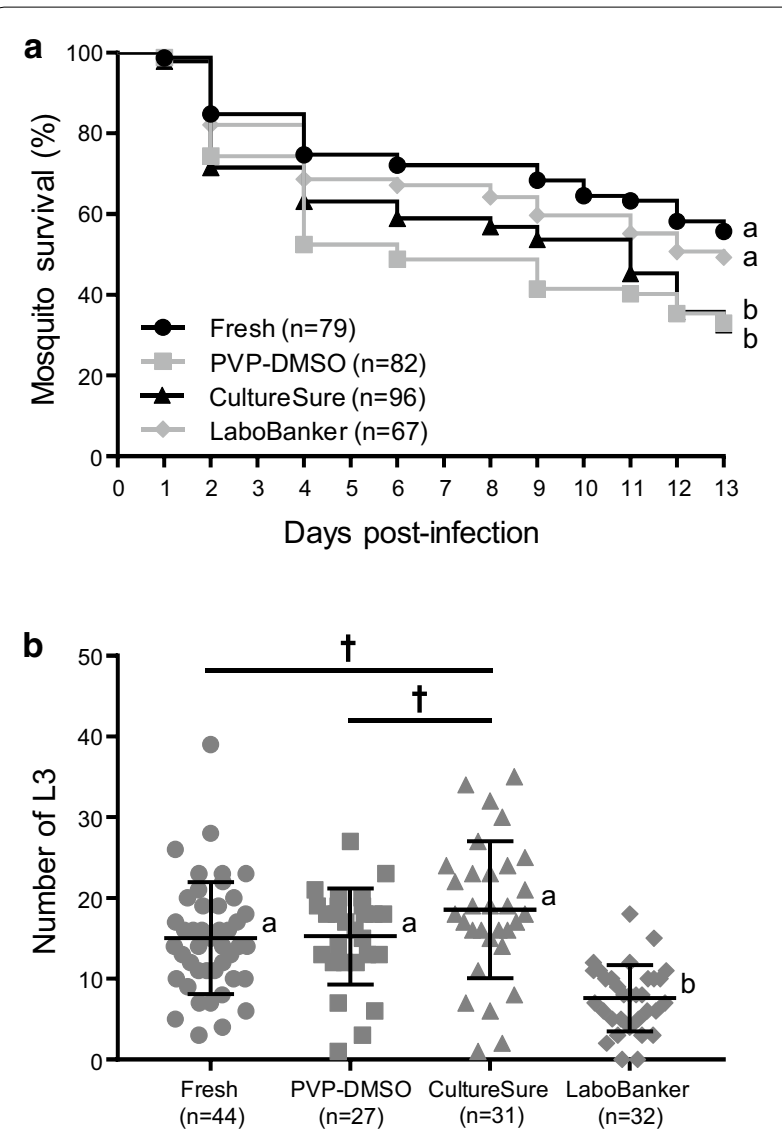

Fig. 3 Analysis of in vivo infectivity of $A$. aegypti mosquitoes with cryopreserved D. immitis microfilariae (mf). a Survival of mosquitoes infected with mf cryopreserved in PVP-DMSO, CultureSure and LaboBanker and non-cryopreserved control (Fresh) over $13 \mathrm{~d}$ post-infection (dpi). The survival rate on 13 dpi was statistically compared using a Chi-square test. Different letters $(a, b)$ indicate significant differences $(P<0.05)$. b The number of third-stage larvae ( $L 3$ ) in the mosquitoes on $13 \mathrm{dpi}$. Each plot shows the $\mathrm{L} 3$ of individual mosquitoes. Error bars $=$ mean \pm SD. The $\mathrm{L} 3$ were statistically compared using Welch's t-test. Significant differences $(P<0.05)$ were observed between different letters $(a, b)$; $†$ indicate marginal differences

the continuous maintenance of an insectary for mosquito production expendable.

Cryopreservation of $D$. immitis $\mathrm{mf}$ has practical applications for veterinary science education [20]. Currently, diagnosis of $D$. immitis infection is usually performed with a commercial antigen detection kit; however, false negatives are common [21]. Adequate skills need to be developed to ensure mf detection in clinical settings. The supply of D. immitis $\mathrm{mf}$ available for veterinary education curricula is limited due to the difficulty of maintaining live animals infected with $D$. immitis $\mathrm{mf}$. We believe our results validate the use of a commercial cryopreservation medium for $D$. immitis $\mathrm{mf}$, overcoming the current issues. 


\section{Conclusions}

In summary, we determined that CultureSure is an optimal commercial cryopreservation solution for the storage of D. immitis mf. CultureSure was successful in cryopreserving purified $\mathrm{mf}, \mathrm{mf}$-infected total blood, and $\mathrm{mf}$ for in vivo mosquito vector experiments. This study will facilitate not only the study of the vector stage of $D$. immitis, but also the study of macrocyclic lactone resistance in heartworms and the consequent education of veterinarians.

\begin{abstract}
Abbreviations
ANOVA: analysis of variance; AR: adjusted residual; DMSO: dimethyl sulfoxide; dpi: days post-infection; dpt: days post-thawing; L3: third-stage larvae; LVP: Liverpool; mf: microfilariae; OUAVM: Obihiro University of Agriculture and Veterinary Medicine; PBS: phosphate buffered saline; PI: propidium iodide; PVP: polyvinylpyrrolidone.
\end{abstract}

\section{Acknowledgements}

We would like to thank R Maeda for providing A. aegypti LVP-OB strain and Editage (http://www.editage.com) for English language editing.

\section{Authors' contributions}

TS and SF designed the experiments. TS, AS and SF carried out the experiments. TS analyzed the data. TS and SF wrote the manuscript, which was edited by AS. SF supervised the study. All authors read and approved the final manuscript.

\section{Funding}

This study was supported by JSPS KAKENHI (Grant numbers $19 \mathrm{H} 03121$ and 19 KK0175 to SF)

\section{Availability of data and materials}

Data supporting the conclusions of this article are provided within the article.

\section{Ethics approval and consent to participate}

This study was carried out in strict accordance with the recommendations in the Guide for Laboratory Animals of OUAVM. The protocol was approved by the committee of the Animal Experiments of the OUAVM (permit number 19-115).

\section{Consent for publication}

Not applicable.

\section{Competing interests}

The authors declare that they have no competing interests.

Received: 16 March 2020 Accepted: 21 July 2020

Published online: 29 July 2020

\section{References}

1. Simón F, Siles-Lucas M, Morchón R, González-Miguel J, Mellado I, Carretón $E$, et al. Human and animal dirofilariasis: the emergence of a zoonotic mosaic. Clin Microbiol Rev. 2012;25:507-44.

2. Webber WA, Hawking F. Experimental maintenance of Dirofilaria repens and D. immitis in dogs. Exp Parasitol. 1955;4:143-64.

3. Weinman D, McAllister J. Prolonged storage of human pathogenic protozoa with conservation of virulence; observations on the storage of helminths and leptospiras. Am J Hyg. 1947:45:102-21.

4. Taylor AE. Studies on the microfilariae of Loa loa, Wuchereria bancrofti, Brugia malayi, Dirofilaria immitis, D. repens and D. aethiops. J Helminthol. 1960;34:13-26.

5. Lok JB, Mika-Grieve M, Grieve RB. Cryopreservation of Dirofilaria immitis microfilariae and third-stage larvae. J Helminthol. 1983;57:319-24.
6. Bartholomay LC, Farid HA, El Kordy E, Christensen BM. Short report: a practical technique for the cryopreservation of Dirofilaria immitis, Brugia malayi, and Wuchereria bancrofti microfilariae. Am J Trop Med Hyg. 2001;65:162-3.

7. Takahashi A, Yamada K, Kishimoto M, Shimizu J, Maeda R. Computed tomography (CT) observation of pulmonary emboli caused by long-term administration of ivermectin in dogs experimentally infected with heartworms. Vet Parasitol. 2008;155:242-8.

8. Vatta AF, Dzimianski M, Storey BE, Camus MS, Moorhead AR, Kaplan RM, et al. Ivermectin-dependent attachment of neutrophils and peripheral blood mononuclear cells to Dirofilaria immitis microfilariae in vitro. Vet Parasitol. 2014;206:38-42.

9. Galal M, Fujimaki Y, Shimada M, Aoki Y. Comparison of the methods available for purification of Brugia pahangi microfilariae in the peritoneal lavage of jirds (Meriones unguiculatus). Trop Med. 1989;31:103-9.

10. Lowrie RC Jr. Cryopreservation of the microfilariae of Brugia malayi, Dirofilaria corynodes, and Wuchereria bancrofti. Am J Trop Med Hyg. 1983;32:138-45.

11. Sharma RD, Bag S, Tawari NR, Degani MS, Goswami K, Reddy MV. Exploration of 2,4-diaminopyrimidine and 2,4-diamino-s-triazine derivatives as potential antifilarial agents. Parasitology. 2013;140:959-65.

12. Ogunrinade A. The measurement of blood meal size in Aedes aegypti (L.). Afr J Med Med Sci. 1980;9:69-71.

13. Jeffery GM. Blood meal volume in Anopheles quadrimaculatus, A. albimanus and Aedes aegypti. Exp Parasitol. 1956;5:371-5.

14. Clark T, Murray JS, Lane P, Politzer P. Why are dimethyl sulfoxide and dimethyl sulfone such good solvents? J Mol Model. 2008;14:689-97.

15. McCall JW, Genchi C, Kramer LH, Guerrero J, Venco L. Heartworm disease in animals and humans. Adv Parasitol. 2008;66:193-285.

16. Hampshire VA. Evaluation of efficacy of heartworm preventive products at the FDA. Vet Parasitol. 2005:133:191-5.

17. Pulaski CN, Malone JB, Bourguinat C, Prichard R, Geary T, Ward D, et al. Establishment of macrocyclic lactone resistant Dirofilaria immitis isolates in experimentally infected laboratory dogs. Parasit Vectors. 2014;7:494.

18. Bowman DD. Heartworms, macrocyclic lactones, and the specter of resistance to prevention in the United States. Parasit Vectors. 2012;5:138.

19. Bourguinat C, Lee AC, Lizundia R, Blagburn BL, Liotta JL, Kraus MS, et al. Macrocyclic lactone resistance in Dirofilaria immitis: failure of heartworm preventives and investigation of genetic markers for resistance. Vet Parasitol. 2015;210:167-78.

20. Long SA, Rhinehart J, Shrake J, Marsh AE. Feasibility and comparative analysis of Dirofilaria immitis microfilaria freezing and fixation for student instruction and assessment of clinical parasitology skills. BMC Vet Res. 2020;16:31.

21. Little SE, Raymond MR, Thomas JE, Gruntmeir J, Hostetler JA, Meinkoth $\mathrm{JH}$, et al. Heat treatment prior to testing allows detection of antigen of Dirofilaria immitis in feline serum. Parasit Vectors. 2014:7:1.

\section{Publisher's Note}

Springer Nature remains neutral with regard to jurisdictional claims in published maps and institutional affiliations.
Ready to submit your research? Choose BMC and benefit from:

- fast, convenient online submission

- thorough peer review by experienced researchers in your field

- rapid publication on acceptance

- support for research data, including large and complex data types

- gold Open Access which fosters wider collaboration and increased citations

- maximum visibility for your research: over $100 \mathrm{M}$ website views per year

At BMC, research is always in progress.

Learn more biomedcentral.com/submissions 